\title{
Estenosis aórtica severa: ¿qué ves cuando me ves?
}

Víctor H. Abregu*

Martín R. González**

Alma S. Arrioja Salazar***

\begin{tabular}{l} 
Correspondencia \\
Víctor H. Abregu \\
1 victorabregu@gmail.com \\
\hline
\end{tabular}

1victorabregu@gmail.com

* Técnico en Prácticas Cardiológicas, sonografista no médico. Sanatorio Trinidad Palermo y San Isidro. Buenos Aires. Argentina

** Licenciado en Producción de Bioimágenes, sonografista no médico. Sanatorio Trinidad San Isidro. Buenos Aires. Argentina

*** Cardiólogo-ecocardiografista. Clínica Dávila. Santiago de Chile. Chile

Recibido: $31 / 05 / 2020$

Aceptado: $31 / 05 / 2020$

En línea: 31/07/2020

Citar como: Abregu VH, González MR, Arrioja Salazar AS. Estenosis aórtica severa: ¿qué ves cuando me ves? Rev Ecocar Pract (RETIC). 2020 (Jul); 3 (2): 45-47. doi: 10.37615/retic.v3n2a14.

Cite this as: Abregu VH, González MR, Arrioja Salazar AS. Echocardiographic evaluation of aortic stenosis. Rev Ecocar Pract (RETIC). 2020 (Jul); 3 (2): 45-47. doi: 10.37615/retic.v3n2a14.

\section{Palabras clave}

$\triangleright$ Estenosis valvular aórtica

$\triangleright$ Válvula aórtica

\section{Keywords}

$\triangleright$ Aortic valve stenosis

$\triangleright$ Aortic valve

\section{RESUMEN}

La estenosis aórtica es la valvulopatía más frecuente en el mundo y su incidencia se incrementa con el envejecimiento de la población. El ecocardiograma es una herramienta útil para la evaluación no invasiva de pacientes con estenosis valvular aórtica en todas sus fases de evolución.

\section{ABSTRACT}

Aortic stenosis is the most frequent valve disease in the world and its incidence increases with the aging of the population. The echocardiogram is a useful tool for the non-invasive evaluation of patients with aortic valve stenosis in all its stages of evolution.

\section{Introducción}

Actualmente, la estenosis aórtica es la valvulopatía más frecuente en el mundo y su incidencia va incrementando con el envejecimiento de la población. La enfermedad degenerativa con calcificación valvular aórtica es la etiología más frecuente, seguida por las enfermedades congénitas y la afección reumática. La disminución progresiva del área valvular aórtica hasta un punto crítico genera incapacidad para incrementar el gasto cardíaco y, en determinadas condiciones, una caída neta del volumen latido con las consecuencias clínicas subsecuentes (síncope, disnea, etc.). La ecocardiografía es una herramienta útil para la evaluación no invasiva de pacientes con estenosis valvular aórtica en todas sus fases de evolución.

\section{Técnica para la evaluación}

La ecocardiografía transtorácica (ETT) es la herramienta de estudio de primera línea, quedando la ecocardiografía transesofágica (ETE) y otras técnicas como la tomografía cardíaca reservadas para cardiólogos entrenados en el área.

Antes de comenzar con el estudio transtorácico, se deberán obtener los signos vitales del paciente, incluyendo presión arterial, ritmo y frecuencia cardíaca, porque si están alterados, se puede sobreestimar o subestimar la valoración de la severidad de la estenosis aórtica (EAo). En el caso de la hipertensión arterial no controlada, por ejemplo, suelen obtenerse valores infraestimados de velocidad y gradientes anterógrados a través de la válvula aórtica (VAo), con la consiguiente sobreestimación del área valvular aórtica (AVA) y subestimación de la severidad real(1).

El siguiente paso es ajustar todos los parámetros técnicos del equipo antes de comenzar el estudio (profundidad, escala de color, velocidad de barrido, PRF, etc.) con la finalidad de obtener las imágenes de mayor calidad posible para emisión de conclusiones.

Para la valoración de la severidad de la EAo hay que usar múltiples parámetros. Las guías de cuantificación valvular(2) recomiendan obtener la velocidad anterógrada transvalvular, gradiente medio y AVA efectiva obtenida por ecuación de continuidad (EC). A pesar de no estar recomendado de primera línea en las guías mencionadas, es recomendable comenzar siempre con la valoración cualitativa de la apertura valvular por modo bidimensional o tridimensional, y utilizar, como se mencionará más adelante, varios parámetros que están en relación con el estudio de la morfología de flujo transvalvular aórtico. La medición directa del área por planimetría (AVA anatómica) en la ETT se debe reservar sólo para los casos con buena definición de los bordes de coaptación y sin alta carga de calcio valvular que deforme la apertura valvular.

Hay que tener en cuenta que deben explotarse todas las ventanas y vistas ecocardiográficas para valorar la VAo, incluyendo la ventana paraesternal derecha, 
subcostal y supraesternal, que no suelen ser rutinarias en algunos laboratorios. Ello se debe a que en muchas ocasiones y dependiendo de la posición anatómica de la VAo y aorta ascendente, los registros Doppler del gradiente pueden quedar subestimados si sólo se valoran las vistas apicales.

El paso en el estudio del paciente se realiza desde la ventana paraesternal izquierda en sus ejes largo y corto (Vídeo 1 y Vídeo 2), donde se valora cualitativamente la morfología de la VAo, la apertura valvular y, con el modo M (Figura 1) se estudia la movilidad y separación de los velos aórticos, que cuando es menor a $8 \mathrm{~mm}$ tiene alta especificidad para EAo severa. La valoración tridimensional con reconstrucción multiplanar (MPR, por sus siglas en inglés) permite obtener una mejor aproximación del AVA anatómica (Figura 2), si bien se reserva para la ETE, donde la imagen valvular es de calidad superior.

En la misma vista de eje largo paraesternal izquierdo se realiza la medición del diámetro del tracto de salida del ventrículo izquierdo (TSVI), por debajo del plano valvular aórtico y lo más adyacente al mismo según las últimas recomendaciones, en mesosístole y guiado por electrocardiograma (Figura 3). Es necesario buscar (si se tiene modo tridimensional es muy útil la herramienta de X-plane) el diámetro mayor del TSVI, ya que una medida incorrecta en el TSVI va a producir errores acumulativos en el cálculo del AVA y, por tanto, un AVA irreal.

Posteriormente, deben evaluarse la velocidad y gradientes transvalvulares aórticos con Doppler continuo (Figura 4), como se mencionaba, en todas las ventanas ecocardiográficas. Se debe tener en cuenta que una incorrecta alineación con el Doppler podría subestimar o sobreestimar las mediciones, por lo que el posicionamiento del transductor y la colocación adecuada de la muestra de volumen del Doppler, así como la experiencia del operador serán de vital importancia para las conclusiones. Debe documentarse en el informe el plano donde se obtuvo el gradiente máximo para facilitar la comparación con estudios posteriores. En el caso de que el ritmo cardíaco del paciente no sea regular se debe obtener un promedio de al menos 10 medidas para tener una cuantificación precisa de una estenosis.

Del espectro transvalvular aórtico obtenido con Doppler continuo se miden la velocidad máxima y media, el gradiente máximo y medio y la integral tiempo-velocidad (ITV). Otros parámetros útiles pueden ser el tiempo de aceleración (TA, tiempo al pico) y el tiempo eyectivo (tiempo que dura el flujo, TE). Se debe recordar que el Doppler continuo obtiene siempre el máximo registro de velocidad y gradientes en el trayecto explorado independientemente del lugar donde se produce la mayor aceleración. En el caso de que existan estenosis seriadas en el TSVI se debe valorar con cuidado el lugar donde se produce la mayor obstrucción. Esta valoración de lesiones seriadas puede ser muy difícil o imposible con la ETT y muchas veces obliga a hacer un estudio transesofágico.

Las recomendaciones de cuantificación de valvulopatías(2) ${ }^{(2)}$ sonsideran que una EAo es severa cuando la velocidad máxima es mayor a $4 \mathrm{~m} / \mathrm{s}$, el gradiente medio superior a $40 \mathrm{mmHg}$ y el área valvular menor a $1,0 \mathrm{~cm}^{2}$. Al trazar el contorno del flujo transvalvular aórtico se obtiene el gradiente máximo, el gradiente medio y la integral velocidad-tiempo. Otros parámetros indicativos de severidad pueden ser el espectro redondeado con ausencia de clicks eyectivos, aumento del TA por encima de $100 \mathrm{~ms}$ ( $94 \mathrm{~ms}$ ofrece mayor especificidad) y el incremento de la relación entre el TA y TE por encima de 0,35(3).

Una vez obtenido el espectro transvalvular aórtico, en la ventana apical de cinco o tres cámaras, se evalúa con Doppler pulsado el flujo en TSVI. Trazando el contorno del flujo se obtendrá la velocidad máxima y media, gradientes máximo y medio, e ITV del TSVI (Figura 5).
Con el diámetro del TSVI, ITV aórtico e ITV del TSVI es posible obtener el AVA mediante la EC (AVA efectiva). El cálculo viene incorporado en el software de análisis y se muestra en el equipo automáticamente al obtener las tres medidas mencionadas. El AVA puede calcularse manualmente con la siguiente fórmula:

$$
\text { Área valvular aórtica }=0,785 \times \text { Diámetro del TSVI }{ }^{2} x \frac{\text { ITV TSVI }}{\text { ITV VAo }}
$$

Un AVA inferior a $1 \mathrm{~cm}^{2}$, una velocidad máxima transvalvular superior a $4 \mathrm{~m} / \mathrm{s}$ y un gradiente medio superior a $40 \mathrm{mmHg}$ indican severidad de la EAo. Adicionalmente, se suele calcular el AVA o cociente adimensional, dividiendo la integral velocidad-tiempo del TSVI entre la ITV del flujo transvalvular aórtico. Un valor menor a 0,25 se correlaciona muy bien con una EAo severa ${ }^{(2)}$.

Finalmente, es imperativo realizar un análisis global, tomando en consideración todas las variables obtenidas y las condiciones de carga bajo las que fueron estudiadas.

\section{Estudio por imagen}

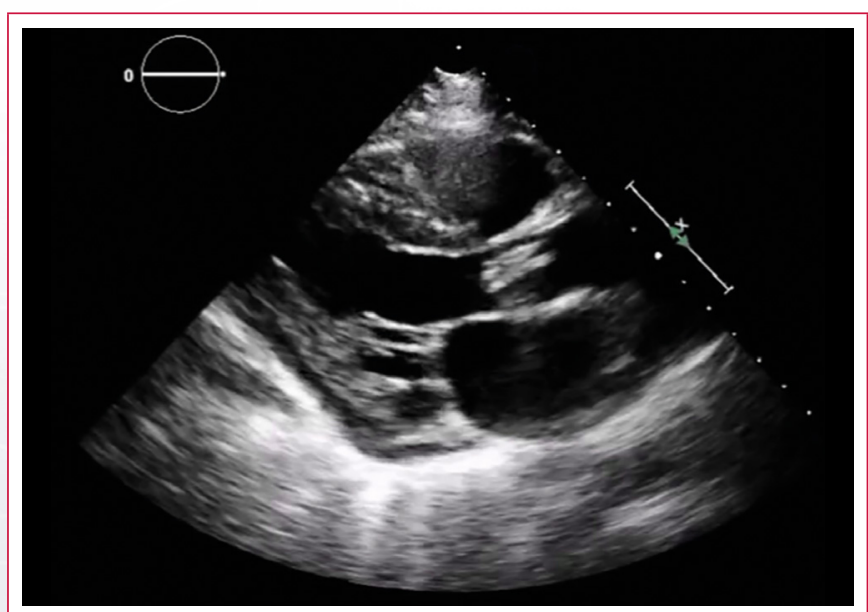

Vídeo 1. ETT, eje largo paraesternal, donde se puede ver la válvula aórtica severamente calcificada con estenosis degenerativa y con limitación importante para su apertura por modo bidimensional

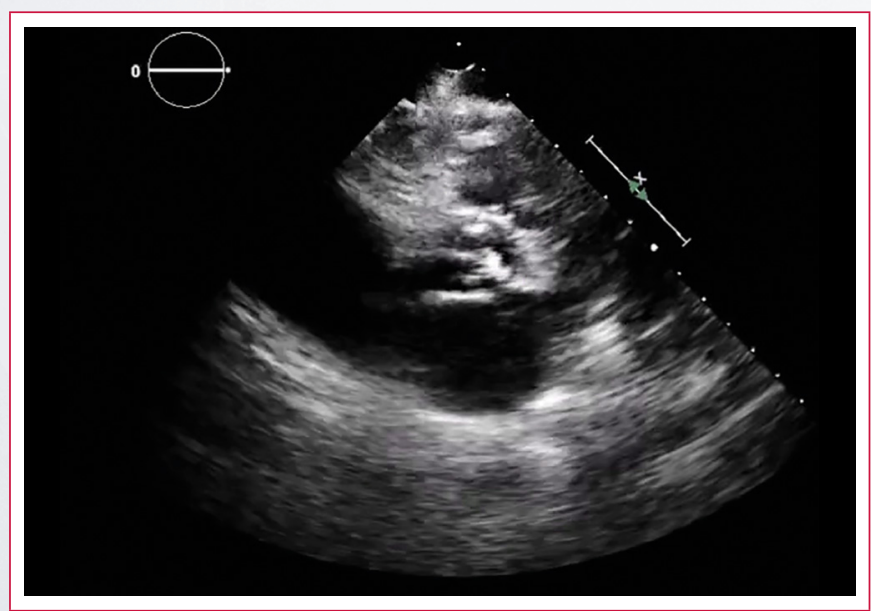

Vídeo 2. ETT, eje corto paraesternal a nivel de grandes vasos. La imagen muestra calcificación avanzada de la válvula aórtica con velos con escaso movimiento 


\section{- Trukipedia truco 01}

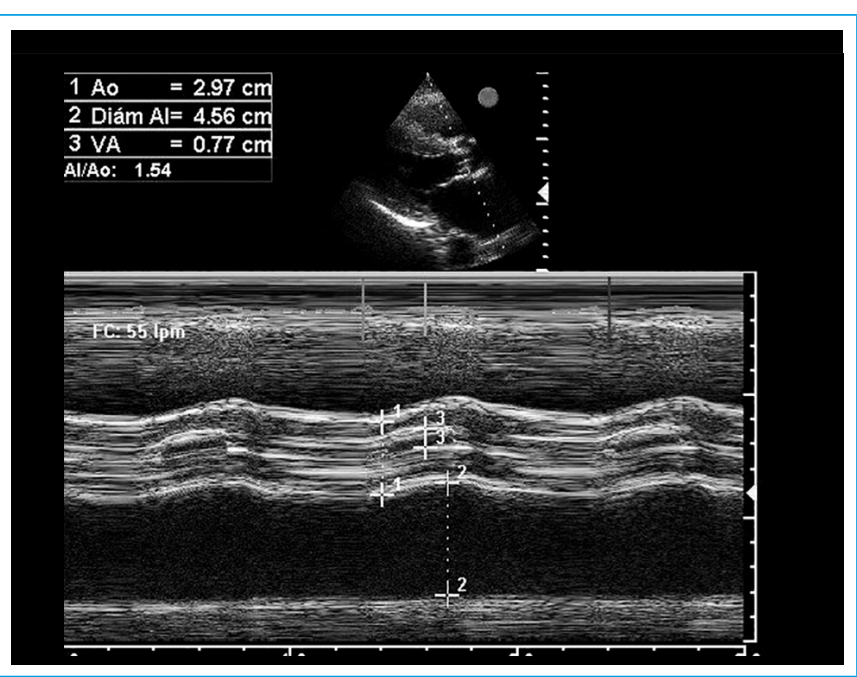

Figura 1. Estudio con modo M del movimiento de los velos aórticos. Una separación inferior a $8 \mathrm{~mm}$ sugiere estenosis importante

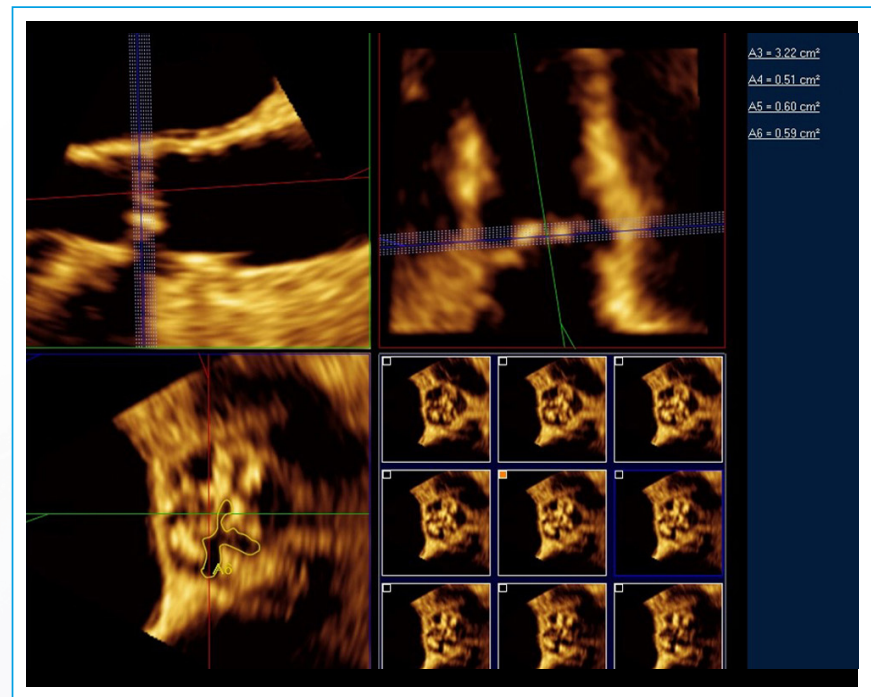

Figura 2. Ejemplo de la medición directa del área valvular aórtica anatómica por planimetría con ecocardiografía tridimensional

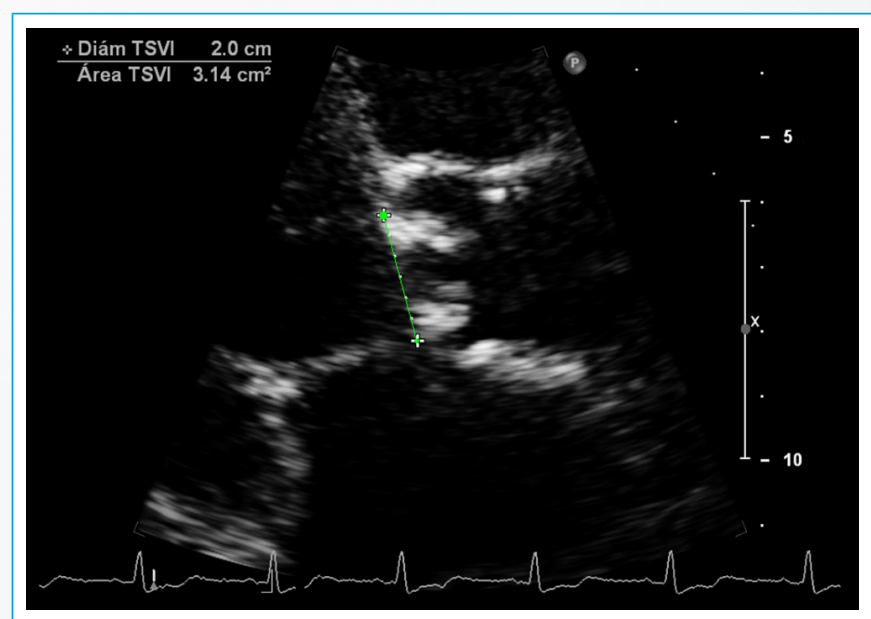

Figura 3. Medida del TSVI. Una medida precisa es más sencilla cuando se tiene una imagen bien centrada y con zoom del área de interés. La medida se debe hacer en mesosístole

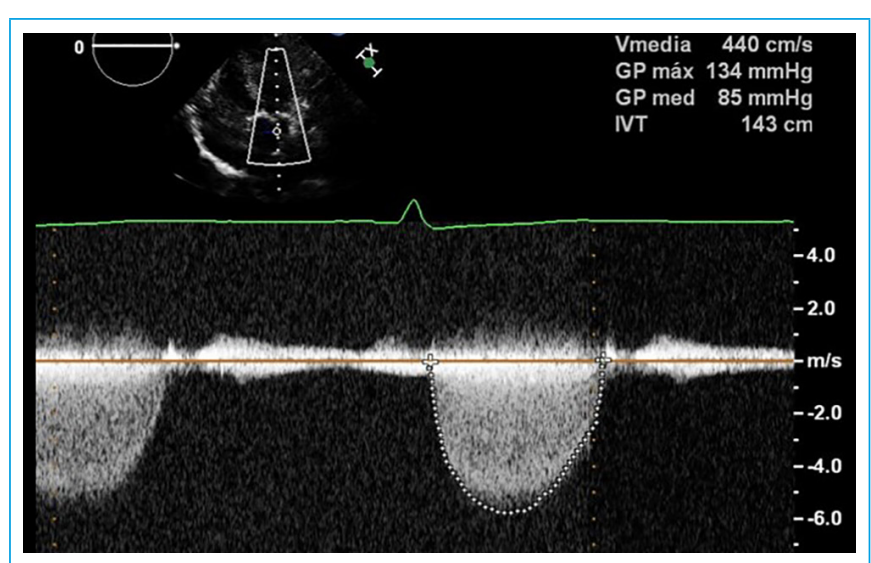

Figura 4. Medida del flujo transvalvular aórtico. Se obtiene la integral tiempo-velocidad, velocidad máxima y gradientes máximo y medio. Las velocidades y gradientes sugieren estenosis severa

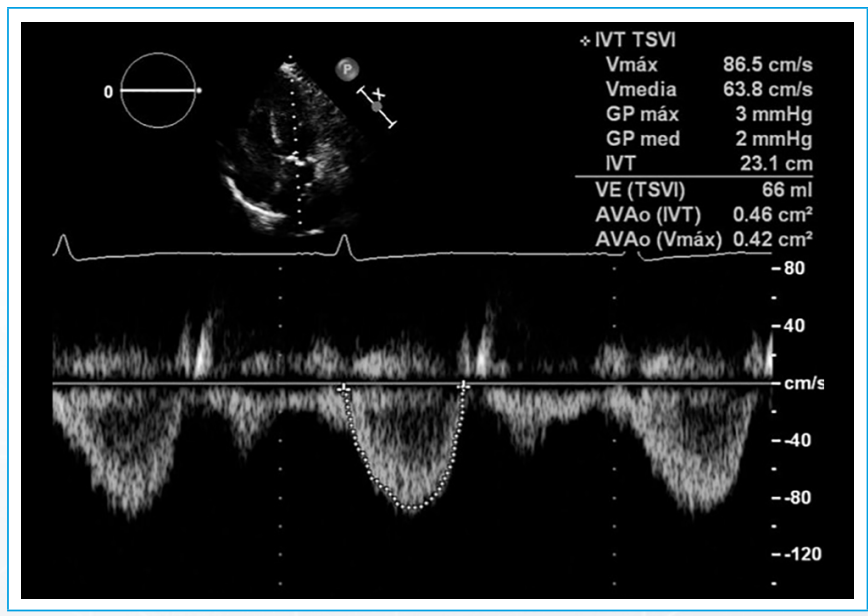

Figura 5. Medida del flujo en TSVI. Se obtiene la integral tiempo-velocidad, velocidad máxima y gradientes máximo y medio. Como ya se tenía hecho el resto de las medidas, el equipo también ha dado automáticamente el área valvular aórtica

\section{Ideas para recordar}

La cuantificación de la severidad de la EAo tiene importancia pronóstica y está basada en la ecocardiografía.

Existen múltiples parámetros que pueden ser útiles, pero que también pueden tener limitaciones debido a múltiples problemas como los puntos de corte establecidos para definir la severidad, los errores en las mediciones y las asunciones geométricas.

- El ecocardiografista debe ser muy cuidadoso para valorar todos los parámetros y poder obtener una impresión global de la situación del paciente.

\section{Bibliografía}

1. Kodem L, Dumesnil JG, Rieu R, et al. Impact of systemic hypertension on the assessment of aortic Stenosis. Heart 2005; 91: 354-361.

2. Baumgartner $\mathrm{H}$, Hung J, Bermejo J, et al. EAE/ASE Recommendations. Echocardiographic assessment of valve stenosis: EAE/ASE recommendations for clinical practice. Eur J Echocardiogr 2009; 10: 1-25.

3. Gamaza-Chulián S, Díaz-Retamino E, Camacho-Freire S, et al. Acceleration time and ratio of acceleration time to ejection time in aortic stenosis: New echocardiographic diagnostic parameters. JASE 2017; 30 (10): 947-955. 